INTERVENTIONAL CARDIOLOGY AND SURGERY

\title{
Subxiphoid pericardiostomy in the management of pericardial effusions: case series analysis of 368 patients
}

\author{
N Becit, Y Ünlü, M Ceviz, C U Koçoğullari, H Koçak, Y Gürlertop
}

Heart 2005;91:785-790. doi: 10.1136/hrt.2004.037499

See end of article for authors' affiliations

Correspondence to Dr Necip Becit, Atatürk Universitesi loj. No 8/7, 25240 Erzurum, Turkey; necipbecit@hotmail.com

Accepted 27 July 2004

\begin{abstract}
Objective: To assess the effectiveness of subxiphoid pericardiostomy in the treatment and diagnosis of pericardial effusions.

Methods: 368 patients who underwent subxiphoid pericardiostomy and tube drainage for cardiac tamponade, moderate to severe pericardial effusion, or suspicious bacterial aetiology were retrospectively analysed. Biopsies of the pericardium and fluid samples for diagnostic tests were obtained from each patient.

Results: The mean age of the patients was 38.4 years, and the male to female ratio was 220:148. The pericardial effusion was classified by echocardiography as severe in $53 \%$ of the patients, moderate in $43 \%$, and mild in $4 \%$. The incidence of cardiac tamponade was $25 \%$. Myocardial injury requiring sternotomy occurred as an operative complication in $0.8 \%$ of the patients and recurrent effusion necessitating further surgical intervention developed in 10\% of patients. Histopathological examination and the polymerase chain reaction of specimens of pericardium and fluid were helpful for establishing a diagnosis in $90 \%$ of patients with malignancy and $92 \%$ of patients with tuberculous pericarditis. The overall 30 day mortality rate was $0.8 \%$. Patients were followed up for at least one year. Pericardial constriction requiring pericardiectomy developed in $3 \%$ of the patients.

Conclusions: Pericardial effusions of various causes can be safely, effectively, and quickly managed with subxiphoid pericardiostomy in both adults and children.
\end{abstract}

$\mathrm{P}$ ericardial effusion is a potentially dangerous condition, as accumulated fluid in the pericardial sac can ultimately lead to cardiac tamponade and fatal shock. ${ }^{1}$ Pericardial effusion is often associated with an underlying disease or condition and the causes of effusion can vary widely. ${ }^{23}$ Pericardial effusion can be relieved by medical treatment, pericardiocentesis through a needle with or without echocardiographic guidance, or by surgical procedures, such as subxiphoid pericardial tube drainage, by creating a pericardial window through a left anterior thoracotomy, or by video assisted thoracoscopic surgery (VATS). ${ }^{14-16}$

We report our clinical experience of using subxiphoid pericardiostomy to treat pericardial effusion.

\section{PATIENTS AND METHODS}

We reviewed the medical records of 368 patients with pericardial effusion who underwent subxiphoid pericardiostomy for the treatment of persistent symptomatic pericardial effusion during the 13 years between 1990 and 2003 in our clinic.

Echocardiography was used for both diagnosis and determining the severity of the effusion. Thus, when the diastolic echo-free space between the left ventricular posterior wall and pericardium was $<10 \mathrm{~mm}$ it was classified as mild, 10-20 mm was classified as moderate, and $>20 \mathrm{~mm}$ was classified as severe pericardial effusion. Cardiac tamponade was defined by clinical and echocardiographic criteria. ${ }^{8}$ The presence of classic tamponade symptoms-tachycardia, dyspnoea, or tachypnoea with clear lungs-or signsincreased systemic venous pressure, hypotension, or pulsus paradoxus-all together or with echocardiographic findings was accepted as cardiac tamponade. Echocardiographic criteria of tamponade were examined by two dimensional and Doppler echocardiography. Two dimensional echocardiographic criteria of tamponade were early diastolic collapse of the right ventricle, late diastolic collapse of the right or left atrium, and plethora of the inferior vena cava with pericardial effusion. Doppler echocardiographic criteria of tamponade were major increases of tricuspid E flow and major decreases of mitral E flow during inspiration compared with apnoea (with the reverse in expiration). In patients with cardiac tamponade, moderate to severe pericardial effusion, or suspicious bacterial aetiology, we performed subxiphoid pericardiostomy and tube drainage.

In 18 patients with a preoperative diagnosis of tuberculosis, a four drug regimen for tuberculosis in adults (isoniazid $300 \mathrm{mg} /$ day and rifampin $600 \mathrm{mg} /$ day for 12 months, pyrazinamide $2 \mathrm{~g}$ /day for three months, and streptomycin $1 \mathrm{~g}$ /day for two months) was started before surgery. Effusion was drained after a three week course of anti-tuberculosis treatment in haemodynamically stable patients. The tuberculosis treatment regimen was continued for 12 months. In patients with a diagnosis of tuberculous pericarditis a four drug regimen for tuberculosis in adults was given for 12 months postoperatively.

The subxiphoid pericardiostomy was performed under general anaesthesia ( $\mathrm{n}=22,6 \%$ ) or local anaesthesia with sedation ( $\mathrm{n}=346,94 \%)$. General anaesthesia was preferred for children and was induced with $1.5 \mathrm{mg} / \mathrm{kg}$ ketamine. Neuromuscular block was achieved with $0.1 \mathrm{mg} / \mathrm{kg}$ vecuronium and anaesthesia maintained with $60 \% \mathrm{~N}_{2} \mathrm{O}, 40 \% \mathrm{O}_{2}$, and $0.5-1.0 \%$ isoflurane. When general anaesthesia was used, the patient's skin was prepared and draped before the induction of anaesthesia to avoid delay if hypotension developed. For local anaesthesia, a 2\% lidocaine solution was injected into the dermal and subdermal layers. Sedation and analgesia were provided by giving $1 \mathrm{mg} / \mathrm{kg}$ ketamine intravenously. After a short midline dermal and subdermal incision was made in the epigastrium, originating from the xiphoid process, additional solution was injected into the rectus abdominus muscle, the periosteum of the xiphisternum, and the retrosternal space. The retrosternal 
prepericardial space was entered by incising the linea alba, splitting the xiphoid process, and dissecting it retrosternally. Dissection of the prepericardial tissues disclosed the lower part of the anterior pericardium. The pericardium was grasped and pulled down, and a piece of anterior pericardium, about $2-4 \mathrm{~cm}$ in diameter, was excised under direct vision and submitted for histopathological analysis. The pericardial cavity was decompressed and samples of the fluid were collected for culture and cytological analysis. To prevent acute cardiac dilatation, during the decompression of the pericardial cavity intravenous digoxin was given and the pericardial cavity was gradually decompressed in patients with chronic severe pericardial effusion. The pericardial cavity was examined under direct vision, by digital examination, or both to detect any tumour or adhesion. Adhesions were gently digitally lysed and loculations were opened to enhance satisfactory drainage as needed. Through a separate stab wound in the left upper abdomen, a soft chest tube was placed in the pericardial cavity lateral to the right ventricle from the pericardiotomy for postoperative suction drainage. It is important to place the tube through a separate incision because a tube left in the operative field may cause wound infection or an incisional hernia. The pericardial incision was left open and the subxiphoid incision was closed. The tube was connected to an underwater seal drainage system. The drainage tube was removed when fluid drainage ceased. Intrapericardial instillation of cytostatic or sclerosing agents was not used after the procedure.

Fifteen patients with mild haemorrhagic effusion and cardiac tamponade caused by trauma or invasive cardiac interventions were considered haemodynamically unstable for surgical subxiphoid pericardiostomy, even under local anaesthesia. For this reason, they underwent pericardiocentesis guided by fluoroscopy in the cardiac catheterisation laboratory with ECG monitoring. Pericardiocentesis provided immediate relief for patients with symptoms and signs of cardiac tamponade. These patients later underwent subxiphoid pericardiostomy for the re-collection of haemorrhagic pericardial effusion.

In patients with both pericardial effusion and pleural effusion, thoracentesis was performed or a chest tube was inserted as required.

All patients were followed up with physical examinations and echocardiography in our outpatient clinic for at least one year after discharge.

\section{Statistical analysis}

Numerical results are given as mean (SD). The $\chi^{2}$ test was used to compare proportions between groups (comparison of the rate of recurrence between patient groups with uraemic pericarditis, idiopathic and undefined pericarditis, tubercu-

\begin{tabular}{|c|c|}
\hline Symptoms & Number of patients \\
\hline $\begin{array}{l}\text { Dyspnoea } \\
\text { Chest pain } \\
\text { Palpitation } \\
\text { Oedema } \\
\text { Cough } \\
\text { Fever } \\
\text { Orthopnoea } \\
\text { Abdominal swelling } \\
\text { Night sweats } \\
\text { Chills } \\
\text { Syncope } \\
\text { Dysphasia }\end{array}$ & $\begin{array}{l}265(72 \%) \\
184(50 \%) \\
192(52 \%) \\
114(31 \%) \\
96(26 \%) \\
92(25 \%) \\
77(21 \%) \\
55(15 \%) \\
48(13 \%) \\
30(8 \%) \\
8(2 \%) \\
8(2 \%)\end{array}$ \\
\hline
\end{tabular}

Table 2 Signs of pericardial effusion in 368 patients

\begin{tabular}{ll}
\hline Temperature ( $\mathrm{C}$ ) & $37.4(0.86)$ \\
Pulse rate (beats/min) & $106(16.7)$ \\
Respiratory rate (breaths/min) & $22(3.4)$ \\
Systolic blood pressure (mm Hg) & $119(15.1)$ \\
Diastolic blood pressure $(\mathrm{mm} \mathrm{Hg})$ & $77(11.9)$ \\
Jugular venous distension $(\mathrm{cm} \mathrm{H}$ ) & $9.7(2.92)$ \\
Paradoxical pulse $(\mathrm{mm} \mathrm{Hg})$ & $8.6(4.75)$ \\
Tachycardia (>100 beats/min) & $240(65 \%)$ \\
Rales & $158(43 \%)$ \\
Oedema & $125(34 \%)$ \\
Friction rub & $92(25 \%)$ \\
Soft first and second heart sounds & $77(21 \%)$ \\
Hepatomegaly & $70(19 \%)$ \\
Kussmaul's sign & $66(18 \%)$ \\
Ascites & $44(12 \%)$ \\
Low arterial blood pressure due to tamponade & $15(4 \%)$ \\
\hline Results are given as mean (SD) or number (\%).
\end{tabular}

lous pericarditis, and malignant processes invading the pericardium). Fisher's exact test was used to compare the constriction rate in patients with tuberculous and nontuberculous bacterial pericarditis. The McNemar test was to compare proportions within one group (to assess the significance of the rates of recurrence and constriction in patients with tuberculous pericarditis). Differences were considered significant if $\mathrm{p}<0.05$.

\section{RESULTS}

The records of 368 patients were reviewed: there were 220 male $(60 \%)$ and 148 female patients $(40 \%)$, whose ages ranged from 5-78 years (mean 38.4 years). All the patients were symptomatic; table 1 lists the presenting symptoms. The predominant symptom was dyspnoea and the main preoperative finding was tachycardia (table 2).

Echocardiographic analysis showed mild effusion in 15 patients $(4 \%)$, moderate effusion in 158 patients $(43 \%)$, and severe effusion in 195 patients (53\%). The symptoms and signs in all 92 patients with cardiac tamponade were nonspecific, but 77 had increased systemic venous pressure, a pulsus paradoxus, and tachycardia despite having normal blood pressure. Only 15 patients had additional hypotension caused by tamponade; they had suffered trauma and had mild pericardial effusion. The causes of pericardial effusion in these cases were blunt injury to the thorax $(n=4)$ and invasive cardiac interventions, including coronary angioplasty, stent implantation, and temporary endocardial pacemaker implantation $(\mathrm{n}=11)$. All the patients with symptomatic pericardial effusion obtained immediate subjective relief from pericardiostomy, which normalised pulse rate and blood pressure; jugular venous distension simultaneously subsided.

Myocardial injury attributable to the operation occurred in three patients $(0.8 \%)$ and could not be controlled by the subxiphoid approach. An immediate median sternotomy was therefore required. Myocardial injury occurred during the first pericardial excision because of severe pericardial adhesions in two patients with recurrent tuberculous pericarditis. The third patient had uraemic pericardial effusion, and right atrial rupture occurred during insertion of the pericardial tube into the pericardial cavity as a result of the rough insertion of a stiff tube. None of these three patients died of this complication.

The causes of pericardial effusion in this study were uraemic pericarditis $(\mathrm{n}=158,43 \%)$, idiopathic and undefined pericarditis $(\mathrm{n}=81,22 \%)$, malignant processes invading the pericardium $(\mathrm{n}=5 \mathrm{l}, 14 \%)$, tuberculous pericarditis $(\mathrm{n}=37,10 \%)$, non-tuberculous bacterial pericarditis $(\mathrm{n}=18,5 \%)$, trauma $(\mathrm{n}=15,4 \%)$, and others 


\begin{tabular}{|c|c|c|c|c|c|}
\hline \multirow[b]{2}{*}{ Cause of pericardial effusion } & \multirow{2}{*}{$\begin{array}{l}\text { Number of } \\
\text { patients } \\
\text { (\% of total) }\end{array}$} & \multirow{2}{*}{$\begin{array}{l}\text { Recurrence } \\
\text { (\% of diagnostic } \\
\text { group) }\end{array}$} & \multirow{2}{*}{$\begin{array}{l}\text { Constriction } \\
\text { (\% of diagnostic } \\
\text { group) }\end{array}$} & \multicolumn{2}{|c|}{ Mortality } \\
\hline & & & & 30 day & 1 year \\
\hline Uraemic pericarditis & $158(43 \%)$ & $22(14 \%)^{*}$ & 0 & 1 & 0 \\
\hline Idiopathic and undefined pericarditis & $81(22 \%)$ & $2(2 \%) \dagger$ & 0 & 2 & 0 \\
\hline $\begin{array}{l}\text { Malignant processes invading the } \\
\text { pericardium }\end{array}$ & $51(14 \%)$ & $2(4 \%) \dagger$ & 0 & 0 & 21 \\
\hline Tuberculous pericarditis & $37(10 \%)$ & $11(30 \%) \ddagger$ & $5(14 \%) \S$ & 0 & 0 \\
\hline Non-tuberculous bacterial pericarditis & $18(5 \%)$ & 0 & $6(33 \%)$ & 0 & 0 \\
\hline Trauma & $15(4 \%)$ & 0 & 0 & 0 & 0 \\
\hline Blunt thoracic injuries & 4 & 0 & 0 & 0 & 0 \\
\hline Invasive cardiac intervention & 11 & 0 & 0 & 0 & 0 \\
\hline Others & $8(2 \%)$ & 0 & 0 & 0 & 0 \\
\hline Total & 368 & $37(10 \%)$ & $11(3 \%)$ & 3 & 21 \\
\hline \multicolumn{6}{|c|}{$\begin{array}{l}\text { * }+\neq \text { In comparison of the rate of recurrence between patient groups by } \chi^{2} \text { test, } p>0.05 \text { between groups with the } \\
\text { same symbol and } p<0.05 \text { between groups with a different symbol; } \$ p<0.03 \text { for the rates of recurrence and } \\
\text { constriction in patients with tuberculous pericarditis by McNemar test; } ₫ p=0.09 \text { versus the constriction rate in } \\
\text { patients with tuberculous pericarditis by Fisher's exact test. }\end{array}$} \\
\hline
\end{tabular}

( $\mathrm{n}=8,2 \%$ ): rheumatoid arthritis in three patients, systemic lupus erythematosus in two, periarteritis nodosa in two patients, and hypothyroidism in one (table 3).

The drainage volume during the operation was 150$4500 \mathrm{ml}$ (mean (SD) $675(22) \mathrm{ml}$ ) and the average drainage volume during the postoperative period was 321 (9) $\mathrm{ml}$ (the average postoperative drainage period being 4.5 (1.3) days). The highest drainage volume was obtained from a patient with uraemia and the lowest from a patient who developed a ruptured right coronary artery during angioplasty and stent implantation. The fluid was transudate in $180(49 \%)$ patients, haemorrhagic in $122(33 \%)$ patients, exudate in $48(13 \%)$ patients, and purulent in $18(5 \%)$.

Histopathological examination of the pericardial specimen, fluid, or both was positive for malignant cells in 46 (90\%) of the 51 patients with pericardial effusion caused by malignant processes invading the pericardium. Examination of the pericardial fluid alone failed to diagnose the cause of pericardial effusion in 18 patients as malignant processes invading the pericardium, but histopathological examination of the pericardial specimen confirmed the diagnosis in all 18 . Of the 46 patients, 21 had lung cancer, 10 had lymphoma, eight had breast cancer, five had leukaemia, and two had malignant thymoma. Histopathological examination of the pericardial fluid and the pericardial specimen did not find any malignant cells in five patients with preoperatively confirmed lung cancer (four cases) and breast cancer (one case).

Only 16 of the 37 patients with tuberculous pericarditis had a preoperative diagnosis of tuberculosis, with a positive purified protein derivative skin test, positive chest radiograph, or acid resistant bacilli in bronchial secretions. Polymerase chain reaction (PCR) analysis in pericardial fluid for the diagnosis of tuberculous pericarditis was positive in 30 $(80 \%)$ of the 37 patients with tuberculous pericarditis. Cytological examination of fluid and pericardial specimens taken during surgery identified the cause of pericardial effusion in $32(86 \%)$ of the 37 patients with tuberculous pericarditis. The diagnosis of tuberculous pericarditis was established by cytological examination of fluid and pericardial specimens in four of the seven PCR negative patients. In the remaining three patients with a preoperative diagnosis of tuberculosis, both PCR analysis in pericardial fluid and cytological examination of fluid and pericardial specimens failed to make a diagnosis. In $34(92 \%)$ of the 37 patients with tuberculous pericarditis, cytological examination of the pericardial fluid and the pericardial specimen confirmed the diagnosis.
The microorganisms identified in cultures of the pericardial fluid from patients with infectious pericarditis were Mycobacterium tuberculosis $(\mathrm{n}=30)$, Pneumococcus species $(\mathrm{n}=7)$, Streptococcus viridans $(\mathrm{n}=5)$, Haemophilus influenzae $(\mathrm{n}=4)$, and Staphylococcus species $(\mathrm{n}=2)$.

Wound infection developed in $19(5 \%)$ patients, which was successfully treated conservatively. The patients were hospitalised for 4-30 days (mean 5.7 days). Intraoperative mortality was $0 \%$ and the overall 30 day mortality (inhospital) was three of $368(0.8 \%)$. Two of these three patients died as a result of low cardiac output, despite inotropic support (dobutamine $5 \mu \mathrm{g} / \mathrm{kg} / \mathrm{min}$ and adrenaline (epinephrine) $3-5 \mu \mathrm{g} / \mathrm{min}$ ) and resuscitative measures. The third patient had inoperable ischaemic heart disease and congestive cardiac failure and died of multiorgan failure.

Pericardial effusion requiring further surgical intervention recurred in $37(10 \%)$ patients between 20-30 days postoperatively. Of the 37 patients with recurrent pericardial effusion, 22 had uraemic pericarditis, 11 had tuberculous pericarditis, two had idiopathic and undefined pericarditis, and two had malignant processes invading the pericardium. The recurrence rate was higher among the patients with tuberculous pericarditis $(30 \%, 11$ of 37$)$ than among those with uraemic pericarditis $(14 \%, 22$ of 158; p < 0.05). Subxiphoid tube drainage and a pleuropericardial window were performed in all these patients with recurrent effusion by making an oblique incision in the epigastrium originating in the left sternocostal angle. After the pleuropericardial window had been made, none of the patients had a recurrence. All surviving patients were followed up for at least one year. During this follow up period, 21 of the 51 patients with malignancy died. Constrictive pericarditis requiring pericardiectomy developed in 11 (3\%) of the 344 surviving patients.

The incidence of constriction requiring pericardiectomy was $14 \%$ (five of 37 ) in patients with tuberculous pericarditis and $33 \%$ (six of 18 ) in patients with non-tuberculous bacterial pericarditis. In these groups, the incidence of constrictive pericarditis was high $(\mathrm{p}<0.05)$. Constrictive pericarditis developed in five of the 11 patients with recurrent tuberculous pericarditis. Pericardiectomy was performed through a median sternotomy in these cases.

\section{DISCUSSION}

The cause of pericardial effusion is often related to underlying conditions such as uraemia, malignancies (such as lung, breast, and ovarian carcinoma, leukaemia, or lymphoma), infections that are usually viral rather than 
bacterial, autoimmune disorders, and myocardial infarction According to some other reports, uraemic pericarditis is a less frequent cause of pericarditis ${ }^{34}$ but in the present series, it is the most common cause. Our hospital admits the majority of patients with renal failure, as it has the biggest dialysis centre in the region. As a result of economic problems, most of these cases of renal failure are not generally well controlled, resulting in the high incidence of uraemic pericardial effusion seen in the present study.

Presentation of pericardial effusion can range from a minimally symptomatic pericardial effusion to a state of complete cardiovascular decompensation. Although pericardial disease is a common entity, pericardial tamponade is often considered to be an unusual presenting feature. Previous studies reported tamponade in $44 \%$ of patients. ${ }^{35} 6$ Cardiac tamponade occurred in $25 \%$ of our patients.

Symptomatic pericardial effusions occur as a result of multiple disease processes and can be treated with many different procedures. Pericardiocentesis, transcutaneous pericardioscopic pleuropericardial window, and subxiphoid pericardial drainage: which method should be used to treat pericardial effusion? Each of these surgical treatments can be effective, depending on clinical factors and the history of the patient. For this reason, the optimal procedure for treatment of these effusions remains controversial, and none of them is optimal for all patients and circumstances. ${ }^{5-16}$ Pericardiocentesis is life saving in cardiac tamponade and indicated in effusions $>20 \mathrm{~mm}$ on echocardiography (diastole). The most serious complications of pericardiocentesis are laceration and perforation of the myocardium and the coronary vessels. Safety was improved with echocardiographic or fluoroscopic guidance. Recent large echocardiographic series reported an incidence of major complications of $1.3-1.6 \%$. In fluoroscopy guided percutaneous pericardiocentesis cardiac perforations occurred in $0.9 \%$, serious arrhythmias in $0.6 \%$, arterial bleeding in $1.1 \%$, pneumothorax in $0.6 \%$, infection in $0.3 \%$, and a major vagal reaction in $0.3 \% .{ }^{8}$ Transcutaneous pericardioscopy is a new diagnostic tool to visualise macroscopical alterations of both the epicardium and the pericardium. Pericardioscopy makes it possible to inspect the pericardial surface, select the biopsy site, and take numerous samples safely. Targeted pericardial or epicardial biopsy during pericardioscopy was particularly useful in the diagnosis of neoplastic pericarditis. Histological analysis of epicardial or pericardial biopsies can establish the diagnosis in patients with neoplastic pericarditis and tuberculosis. Diagnosis of viral pericarditis can be established by PCR techniques with much higher sensitivity and specificity than viral isolation from fluid and tissue. No major complications occurred in any of the flexible pericardioscopy studies. ${ }^{89}$ The pleuropericardial window on VATS is better in chronic pericardial effusion (for infective or systemic disease) and in recurrence after subxiphoid drainage. ${ }^{58912}$ Surgical drainage is preferred in traumatic haemopericardium and purulent pericarditis. ${ }^{5-16}$ The major complication rate was $0.8 \%$ in our study and it is lower than that of pericardiocentesis.

The ideal procedure should be easy to perform, result in minimal morbidity and mortality, ensure complete and permanent drainage, have infrequent recurrences, and provide sufficient histological, cytological, and microbiologic specimens for diagnosis of the cause of the effusion. The two primary modalities used to drain symptomatic pericardial effusions are transcutaneous pericardioscopy and open subxiphoid surgical drainage. The potential advantages of pericardioscopy and catheter drainage are no need for an incision and less resultant pain, visualisation of macroscopic alterations of both the epicardium and the pericardium, selection of the biopsy site, the ability to take numerous samples safely, and no need for general anaesthesia. But the success rate and feasibility of this procedure are low in patients with small effusions or posteriorly located effusions, and in such conditions a great deal of experience is needed. The potential advantages of subxiphoid pericardiostomy are direct visualisation and exploration of the pericardium and pericardial cavity, the ability to probe the pericardial cavity to allow for complete drainage, biopsy of the pericardium for pathological analysis, and placement of a larger calibre tube for better drainage. ${ }^{7-10}$ Video assisted transthoracic pericardial drainage has been touted as effective for preventing effusion recurrence through a large pericardial resection with the creation of a "pericardial window". ${ }^{5}$ It requires, however, general anaesthesia and single lung ventilation, procedures that are difficult in critically ill patients. ${ }^{5}$ Furthermore, the concept of a pericardial window for permanent drainage of pericardial effusion into the pleural space or peritoneum is misleading because the hole created is quickly sealed by surrounding tissue. ${ }^{5}{ }^{12}$

In our series of 368 patients with pericardial effusion, subxiphoid pericardiostomy was performed under local anaesthesia with sedation $(\mathrm{n}=346,94 \%)$ or general anaesthesia $(n=22,6 \%)$ during the 13 years between 1990 and 2003. General anaesthesia was preferred for children. Pericardiocentesis was performed as a temporary procedure on 15 of our 368 patients. These patients had life threatening haemodynamic instability caused by trauma and subsequently underwent subxiphoid pericardiostomy. Pericardiocentesis was not used electively as a diagnostic and treatment modality for several reasons. Firstly, its safety was considered. Despite reports of high success and low complication rates with echocardiography guided pericardiocentesis, ${ }^{6-11}{ }^{14}{ }^{15}$ we think these figures are probably valid only for the most experienced cardiologists. Secondly, pericardiocentesis should be performed primarily on patients with haemodynamic instability. Thirdly, it has incomplete diagnostic effectiveness in patients with tuberculous pericarditis and purulent and malignant processes invading the pericardium because a pericardial biopsy specimen cannot be taken. As a result, although pericardiocentesis may provide temporary relief to patients with symptoms and signs of cardiac tamponade, it is not adequate for definitive treatment.

Our approach to patients with uraemic pericardial effusion who are usually followed up in our hospital is usually aggressive haemodialysis. In these patients, subxiphoid pericardiostomy is not commonly required, mostly because of suspicious bacterial aetiology. The patients with pericardial effusion included in this study are mostly followed up by other hospitals and they are generally not well controlled. In these patients, we prefer subxiphoidal pericardiostomy for full drainage of the fluid and we send them to the initial centre for chronic haemodialysis after their status becomes stable.

Pericardial effusion requiring further surgical intervention recurred in $37(10 \%)$ patients. Of these, 22 had uraemic pericarditis and were referred to our centre from peripheral hospitals. Thus, the recurrence rate of effusion in the present study was higher than that reported in the literature. ${ }^{8}$. Subxiphoid tube drainage and a pleuropericardial window were performed in all these patients with recurrent effusion by making an oblique incision in the epigastrium originating in the left sternocostal angle. After the pleuropericardial window had been made, none of the patients had a recurrence. We did not prefer to create a pleuropericardial window on VATS to avoid general anaesthesia and single lung ventilation. We did not perform transcutaneous pericardioscopy because of a lack of technical support.

There are no randomised trials comparing the efficacy and safety of systemic versus intrapericardial treatment 
modalities in neoplastic pericardial disease. Systemic antineoplastic drugs as baseline treatment and pericardiocentesis to relieve symptoms, establish diagnosis, and enable intrapericardial instillation of cytostatic or sclerosing agent is the common approach. Recurrence, observed in $40-70 \%$ of patients with large malignant pericardial effusion, may be prevented by intrapericardial instillation of sclerotic agents, cytotoxic drugs, immunomodulators, systemic antitumour treatment, radiation therapy, percutaneous balloon pericardiotomy, or surgical methods. ${ }^{17}{ }^{18}$ In our patients with large malignant pericardial effusion, intrapericardial instillation of cytostatic or sclerosing agents was not used after the procedure, and systemic antineoplastic drugs were given as baseline treatment.

Allen and colleagues ${ }^{13}$ reported on a series of 117 patients with cardiac tamponade resulting from pericardial effusion. The mortality in 94 patients who underwent subxiphoid pericardiostomy was $0 \%$, the complication rate was $1.1 \%$ (one of 94), and the recurrence rate was $1.1 \%$ (one of 94). Conversely, in 23 patients who underwent percutaneous catheter drainage guided echocardiographically by a cardiologist, the mortality was $4.3 \%$ (one of 23 ), the complication rate was $17 \%$ (four of 23 ), and the recurrence rate was $32 \%$ (seven of 22). These authors stated that percutaneous catheter drainage, while less invasive, is associated with increased morbidity, mortality, and effusion recurrence rates. $^{13}{ }^{14}$ Furthermore, the procedure does not include visualisation or biopsy of the pericardium. ${ }^{13}$ In a series of 63 patients with cardiac tamponade undergoing primary pericardiocentesis, Bastian and colleagues ${ }^{14}$ reported a success rate of $81 \%$ and a recurrence rate of $19 \%$. In a similar study, Vayre and colleagues ${ }^{15}$ reported a major complication incidence of $10 \%$, and emergency surgical drainage was required for a failed procedure in $4 \%$ of patients. Late surgical drainage was required for persistence or recurrence of the effusion in $15 \%$ of patients. Pericardiocentesis or percutaneous tube drainage may be a useful temporary treatment for patients with acute tamponade. ${ }^{12} 1415$

Cegielski and colleagues ${ }^{19}$ reported that PCR was positive in 14 of 20 patients with tuberculous pericarditis. In the present study, a definite diagnosis was established by PCR in 30 of 37 patients with tuberculosis. In a series of 38 patients with pericardial effusion reported by Fernandes and colleagues, ${ }^{20}$ a biopsy defined the cause in only four patients (11\%) (as tuberculosis in two and neoplasias in two). In our series of 368 patients, histopathological examination of the pericardial specimen, fluid, or both confirmed the diagnosis in 46 (90\%) of the 51 patients with pericardial effusion caused by malignant processes invading the pericardium. Cytological examination of the pericardial fluid and the pericardial specimen confirmed the diagnosis in 34 (92\%) of the 37 patients with tuberculous pericarditis. We believe that pericardiostomy may be useful for establishing the etiological diagnosis in patients with pericardial effusion, especially in those with tuberculous pericarditis or malignancy.

Palatianos and colleagues ${ }^{21}$ reported that microorganisms were cultured in seven (88\%) of eight patients with exudative pericardial effusion by microbiological processing of the pericardial fluid, whereas in our study, microorganisms were cultured from $48(73 \%)$ of 66 patients with exudative pericardial effusion. Viral pericarditis probably caused pericardial effusion in some of our 81 patients with idiopathic and undefined pericarditis because viruses are not cultured in our microbiology laboratory.

Constrictive pericarditis results from a thickened, scarred, and often calcified pericardium that limits diastolic ventricular filling. Pericardiectomy is commonly performed through a median sternotomy, although some surgeons prefer access through a thoracotomy. Despite a decline, the risk of mortality remains at about $6-19 \% .^{3}$ Heavy calcification and involvement of the visceral pericardium increase the risk. Left ventricular systolic dysfunction may occur after decorticating a severely constricted heart. Prevention of pericardial constriction consists of appropriate treatment of acute pericarditis and adequate pericardial drainage. ${ }^{3}$ In the present study, pericardial constriction requiring pericardiectomy developed in only $3 \%$ of the surviving patients. We conclude that subxiphoid pericardial drainage is effective for treatment of pericardial effusion. The number of patients with constriction in our study was too small for statistical evaluation, but rates of recurrent effusion and constriction are higher in patients with tuberculous pericarditis and other bacterial pericarditis. For this reason, we recommend close follow up of these patients after the first episode.

In conclusion, we believe that subxiphoid pericardiostomy is a safe and effective technique not only to manage patients with pericardial effusion but also to help establish the aetiological diagnosis, especially in patients with tuberculous and malignant processes invading the pericardium.

\section{ACKNOWLEDGEMENTS}

We thank Professor Ömer Akbulut for his help in statistical evaluations.

\section{Authors' affiliations}

N Becit, Y Ünlü, M Ceviz, C U Koçoğullari, H Koçak, Department of Cardiovascular Surgery, Atatürk University School of Medicine, Erzurum, Turkey

Y Gürlertop, Department of Cardiology, Atatürk University School of Medicine

\section{REFERENCES}

1 Sagrista-Sauleda J, Merce J, Permanyer-Miralda G, et al. Clinical clues to the causes of large pericardial effusions. Am J Med 2000;109:95-101.

2 Hoit BD. Management of effusive and constrictive pericardial heart disease. Circulation 2002; 105:2939-42.

3 Thummler F, Schmidt H, Evequoz D. Pericardial effusion in the hospitaldiagnosis and therapy. Schweiz Rundsch Med Prax 1999:23:1573-80.

4 Becit N, Ozyazicioglu A, Ceviz M, et al. Clinical experience with subxiphoid pericardiostomy in the management of pericardial effusions: a study of 240 cases. J Int Med Res 2003;31:312-7.

5 Sugimoto JT, Little AG, Ferguson MK, et al. Pericardial window: mechanisms of efficacy. Ann Thorac Surg 1990;50:442-5.

6 Lindenberger $M$, Kjellberg $M$, Karlsson $E$, et al. Pericardiocentesis guided by 2-D echocardiography: the method of choice for treatment of pericardial effusion. J Intern Med 2003;253:411-7.

7 McDonald JM, Meyers BF, Guthrie TJ, et al. Comparison of open subxiphoid pericardial drainage with percutaneous catheter drainage for symptomatic pericardial effusion. Ann Thorac Surg 2003;76:811-5.

8 Maisch B, Seferovic PM, Ristic AD, et al. Guidelines on the diagnosis and management of pericardial diseases. Eur Heart $J$ 2004; 25:587-610.

9 Maisch B, Ristic AD. Practical aspects of the management of pericardial disease. Heart 2003;89:1096-103.

10 Buchanan CL, Sullivan VV, Lampman R, et al. Pericardiocentesis with extended catheter drainage: an effective therapy. Ann Thorac Surg 2003;76:817-20.

11 del Barrio LG, Morales JH, Delgado C, et al. Percutaneous balloon pericardial window for patients with symptomatic pericardial effusion. Cardiovasc Intervent Radiol 2002;25:360-4.

12 Mazzucotelli J. [Pericardial drainage: the right indication for the right technique]. Presse Med 1997;26:1051-2.

13 Allen KB, Faber LP, Warren WH, et al. Pericardial effusion: subxiphoid pericardiostomy versus percutaneous catheter drainage. Ann Thorac Surg 1999;67:437-40.

14 Bastian A, Meissner A, Lins M, et al. Pericardiocentesis: differential aspects of a common procedure. Intensive Care Med 2000;26:572-6.

15 Vayre F, Lardoux $\mathrm{H}$, Chikli $\mathrm{F}$, et al. [Evaluation of echo-guided pericardiocentesis in cardiac tamponade]. Arch Mal Coeur Vaiss 1998;91:13-20.

16 Tanaka H, Fujita T, Endoh Y, et al. Pericardial tamponade type injury: a 17year study in an urban trauma center in Japan. Surg Today 1999;29:1017-23.

17 Maisch B, Ristic AD, Pankuweit S, et al. Neoplastic pericardial effusion: efficacy and safety of intrapericardial treatment with cisplatin. Eur Heart J 2002;23:1625-31. 
18 Maisch B Ristic AD Pankuweit S Intrapericardial treatment of autoreactive pericardial effusion with triamcinolone: the way to avoid side effects of systemic corticosteroid therapy. Eur Heart J 2002;23:1503-8.

19 Cegielski JP, Devlin BH, Morris AJ, et al. Comparison of PCR, culture, and histopathology for diagnosis of tuberculous pericarditis. J Clin Microbiol 1997:35:3254-7.
20 Fernandes $F$, lanni BM, Arteaga E, et al. Value of pericardial biopsy in the etiologic diagnosis of pericardial diseases. Arq Bras Cardiol 1998;70:393-5.

21 Palatianos GM, Thurer RJ, Pompeo MQ, et al. Clinical experience with subxiphoid drainage of pericardial effusions. Ann Thorac Surg $1989 \cdot 48 \cdot 381-5$

\section{IMAGES IN CARDIOLOGY}

Severe mitral regurgitation caused by annular abscess fistulating into the left atrium

A

14 year old girl was referred for management of Staphylococcus aureus endocarditis, severe mitral regurgitation, and congestive heart failure. She had received chemotherapy for left femoral osteosarcoma diagnosed four months earlier. Transthoracic echocardiography showed a large pericardial effusion and a $20 \times 5 \mathrm{~mm}$ sausage shaped vegetation on the posterior mitral leaflet with severe mitral regurgitation (upper panels). Following pericardiocentesis, and antibiotic and diuretic treatment, the patient underwent mitral valve surgery. Intraoperative transoesophageal echocardiography showed a ruptured abscess cavity measuring $30 \times 18 \mathrm{~mm}$ in the posterior mitral annulus, with regurgitation through the ring abscess (middle and lower panels). At surgery, there was severe panpericarditis and a large posteromedial mitral annular abscess, detaching P2 and P3 scallops from the annulus. The apparent vegetation was in fact the posterior mitral leaflet attached to the dehisced roof of the abscess cavity. The annulus was debrided and reconstructed using an equine pericardial patch and polypropylene sutures, and a $27 \mathrm{~mm}$ St Jude Medical mechanical prosthesis inserted. Transoesophageal echocardiography showed no further regurgitation and the patient is free of cardiac symptoms one year after surgery.

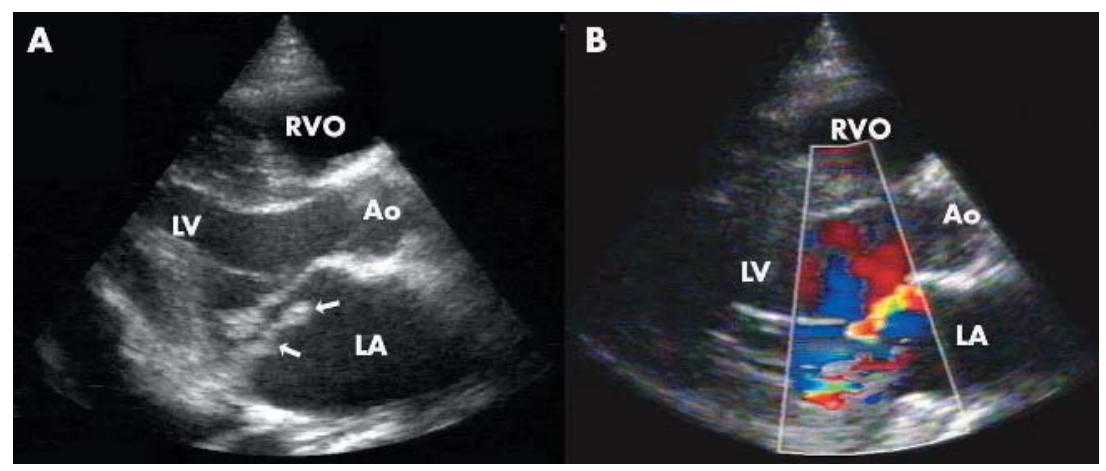

Transthoracic parasternal long axis echocardiograms. (A) Suspected infective vegetation (arrows) attached to a flail posterior mitral leaflet. Ao, aortic root; LA, left atrium; LV, left ventricle; RVO, right ventricular outflow. (B) Anteriorly directed colour jet of mitral regurgitation.

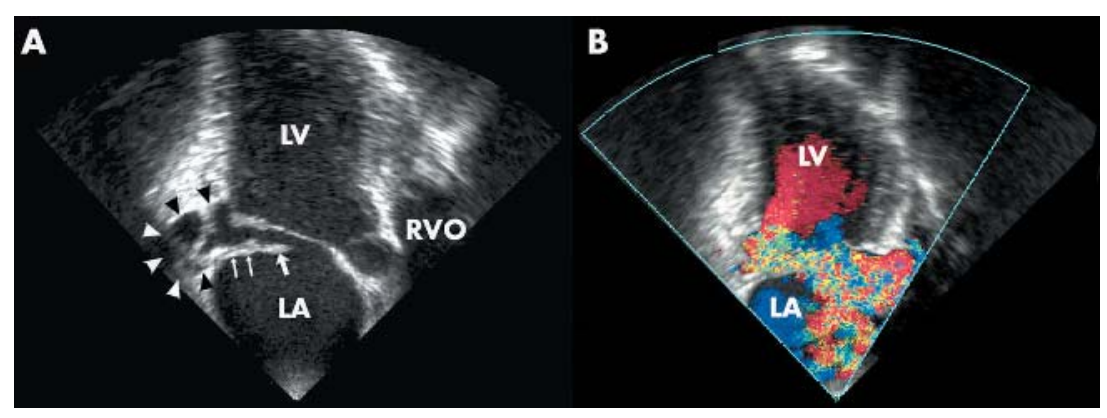

$H$ Yang

L H Ling mdcllh@nus.edu.sg

Transoesophageal long axis echocardiograms. (A) Loculated abscess in the posterior mitral annulus (arrowheads). The presumed vegetation consisted of the posterior valve leaflet (thick arrow) attached to the detached roof of the abscess (slender arrows). (B) Severe regurgitation from the left ventricular to left atrial fistula created by rupture of the abscess.

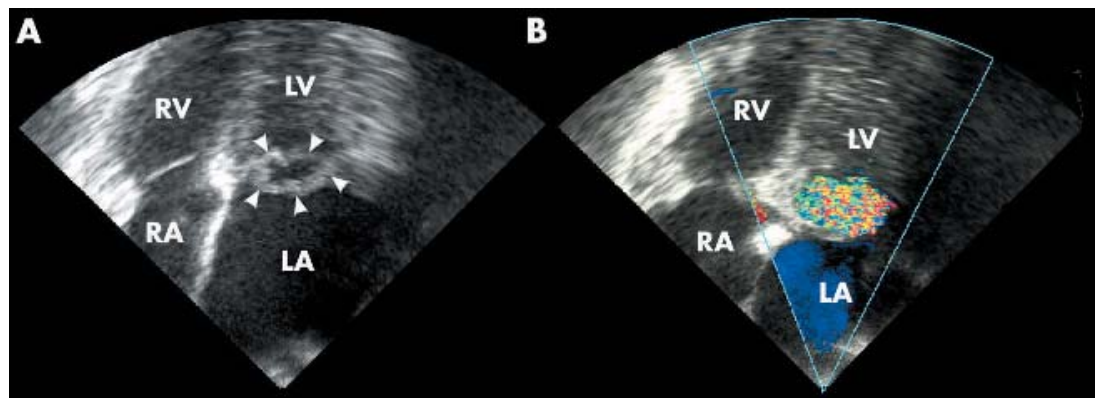

Transoesophageal echocardiograms, four chamber view. (A) Mitral annular abscess seen "enface" (arrowheads). RA, right atrium; RV, right ventricle. (B) Turbulent systolic colour flow within the abscess cavity. 